\title{
Nathalie Roseau, Marie Thébaud-Sorger (dir.), L'emprise du vol. De l'invention à la massification : histoire d'une culture moderne
}

Vérone, MétisPresses, 2013

Larissa Zakharova

\section{OpenEdition}

\section{Journals}

Édition électronique

URL : https://journals.openedition.org/artefact/7358

DOI : 10.4000/artefact.7358

ISSN : 2606-9245

Éditeur :

Association Artefact. Techniques histoire et sciences humaines, Presses universitaires du Midi

Édition imprimée

Date de publication : 18 juin 2015

Pagination : 239-241

ISBN : 978-2-271-08155-1

ISSN : 2273-0753

Référence électronique

Larissa Zakharova, « Nathalie Roseau, Marie Thébaud-Sorger (dir.), L'emprise du vol. De l'invention à la massification : histoire d'une culture moderne », Artefact [En ligne], HS 01 | 2015, mis en ligne le 28 avril 2021, consulté le 05 mai 2021. URL : http://journals.openedition.org/artefact/7358 ; DOI : https:// doi.org/10.4000/artefact.7358

Ce document a été généré automatiquement le 5 mai 2021.

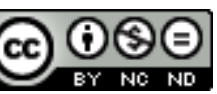

Artefact, Techniques, histoire et sciences humaines est mise à disposition selon les termes de la Licence Creative Commons Attribution - Pas d'Utilisation Commerciale - Pas de Modification 4.0 International. 


\title{
Nathalie Roseau, Marie Thébaud- Sorger (dir.), L'emprise du vol. De l'invention à la massification : histoire d'une culture moderne
}

Vérone, MétisPresses, 2013

\author{
Larissa Zakharova
}

\section{RÉFÉRENCE}

Nathalie Roseau et Marie Thébaud-Sorger (dir.), L'emprise du vol. De l'invention à la massification : histoire d'une culture moderne, Vérone, MétisPresses, 2013, 192 p.

Dans son ouvrage Sociology beyond societies, le sociologue anglais John Urry affirme que la mobilité dans le monde moderne et postmoderne a fait disparaitre les sociétés en tant qu'entités et catégories d'analyse opérantes ${ }^{1}$. Le vol et l'aéronautique ont révolutionné la mobilité des individus, en accélérant la vitesse des déplacements et en permettant de vaincre, au moins en partie, le temps et l'espace. Les déplacements dans l'espace aérien et le franchissement des grandes distances en un temps record grâce aux techniques aéronautiques minimisent l'importance des frontières et font du social un ensemble mouvant et peu structuré. En même temps, l'espace aérien et les mobilités restent toujours l'objet d'une stricte réglementation de la part des États. Le partage de l'espace aérien leur permet d'exercer leur domination politique dans les zones d'influence. Les techniques aéronautiques constituent ainsi de véritables défis politiques dans la mesure où elles participent aux jeux de domination géopolitiques. Le livre dirigé par Nathalie Roseau et Marie Thébaud-Sorger examine les effets contradictoires de l'invention des techniques du survol à travers l'analyse des impacts de l'aéronautique sur les cultures et la perception de l'espace par les individus. 
2 Organisé autour de cinq parties, il multiplie les points de vue afin d'appréhender l'espace aérien et ses techniques. Sont articulées les perspectives sociologique et politique, transnationale et nationale, globale et locale, celle qui voit la tendance à l'unification et à l'intégration et celle qui est, au contraire, attentive à la division et à la structuration du monde. Dans un sens, les techniques aéronautiques, en tant que vecteurs de la mobilité des individus, contribuent à la disparition des sociétés ; dans l'autre, elles sont investies par les gouvernements en tant que moyens de contrôle des espaces et de délimitation précise des sphères d'influence politique. L'avion permet d'accéder aux territoires où les chemins de fer n'existent pas. Dans son article sur les visions politiques et les imaginaires nationaux dans la Norvège de l'entre-deux-guerres, Rune Hovd s'intéresse au rôle de l'aviation dans l'intégration des territoires reculés dans l'ensemble national et dans la sortie de la Norvège de la périphérie européenne. Les techniques aéronautiques contribuent au changement du rapport entre le centre et la périphérie grâce à la réduction de la distance et au réagencement de l'espace. De la même manière, le survol, en tant qu'un acte constitutif des politiques d'aménagement, permet un ancrage territorial, la conquête et la maîtrise du territoire. En atteste la photographie aérienne, comme le montre Frédéric Pousin dans son étude de l'aménagement du littoral aquitain entre 1967 et 1988. Les images prises du ciel font preuves de la complicité entre les technologies du vol et de l'image. Le survol transforme en profondeur les modalités du regard, de la connaissance, de l'écriture et de la peinture, en permettant une vue " totalisante » de la réalité. C'est ce qui ressort de l'article de Teresa Castro sur le tournage des films documentaires sur les champs de bataille de la Première Guerre mondiale en France et en Belgique, et de celui de Gilles Chamerois qui explore l'éthique d'une réversibilité du regard dans des œuvres littéraires et plastiques.

3 L'articulation entre le sociologique et le politique se situe au niveau des perceptions des techniques aéronautiques qui peuvent être façonnées par différents régimes politiques ou par les artistes et peuvent donc se distinguer les unes par rapport aux autres. Dans son chapitre sur l'air comme matière, Andrea Urlberger se penche sur les expérimentations artistiques autour de la matérialité de l'air. L'idée héroïque du vol est tournée en dérision par les objets ordinaires du quotidien auxquels les artistes confèrent la capacité de voler. Le phénomène du patriotisme en tant qu'enjeu politique est intimement lié à l'apparition de héros aviateurs qui deviennent l'expression et l'incarnation de la grandeur et du prestige politique des nations. La maîtrise des techniques et les exploits aériens font naître de nouvelles figures non seulement de masculinité mais aussi de féminité, comme l'exposent Claude d'Abzac-Epezy et Guillaume de Syon dans leurs études des héros de l'air dans la première moitié du $\mathrm{xx}^{\mathrm{e}}$ siècle et des passions et des peurs de l'aviation au travers de la carte postale entre 1890 et 1914. À côté des aviateurs et aviatrices, les inventeurs incarnent également la gloire et le prestige national. Certains pays réécrivent l'histoire des inventions aéronautiques pour s'attribuer la gloire d'être pionniers dans ce domaine fortement stratégique. Ce patriotisme aérien favorise le climat de compétition entre les nations dont la dimension politique est particulièrement forte avec le déclenchement de la Guerre froide.

4 Aux figures des héros s'opposent celles d'antihéros terroristes. La question de la sécurité et de la menace du terrorisme apparaît en filigrane dans les articles de Vanessa R. Schwartz et de Nathalie Roseau, consacrés aux conceptions des aéroports depuis le 
Jet Age. La sécurité en tant qu'enjeu politique national et transnational concerne non seulement la prévention des attaques et les dispositifs de surveillance et de contrôle dans les aéroports, mais aussi la performativité des machines et la garantie contre les chutes et les catastrophes aériennes. La contribution de Caroline Moricot pose la question de la distribution des responsabilités pour la sécurité du vol entre l'homme et la machine. Avec les glass cockpits et le Flight management system, nous sommes face au problème du volontarisme des techniques qui seraient capables de s'opposer aux gestes des hommes. La disparition du risque de l'erreur humaine soulève le problème de la maitrise d'une technique qui n'est plus assimilée à une emprise de l'homme sur la machine, mais qui se résume à la recherche d'un équilibre entre la confiance et la méfiance. De là émerge la question des compétences : dans le contexte de cette innovation technique, les compétences sont repensées et reformulées. Autrement dit, il s'agit de la question de l'impact de l'innovation technique sur les professions et les métiers, comparable avec ce qui se passe dans la téléphonie avec l'avènement de la numérisation.

5 Les techniques de la maîtrise de l'espace traversent les frontières nationales sous l'impulsion de leurs promoteurs qui sont guidés par des objectifs politiques, de concurrence ou de prestige. Dans la compétition entre Boeing et Airbus, c'est la concurrence entre l'Europe et les États-Unis qui se joue. La contribution de David Burigana montre que, par le biais du projet Airbus, l'Europe cherche à s'imposer vis-àvis des États-Unis. Le processus d'intégration européenne va ainsi de pair avec l'innovation technique, même si le projet Airbus correspond à une partie de l'Europe qui ne coïncide pas avec l'Europe de la CEE-UE. Il s'agit d'une coopération intergouvernementale, présentée comme un projet phare de la nouvelle Europe. La présence de moteurs américains dans l'Airbus est alors tue, silence qui rappelle d'autres épisodes de l'histoire de la concurrence économique et technique, lorsque l'Union soviétique construisait à partir de prototypes américains des avions aux noms différents, puis les présentait comme des preuves de la prouesse technique nationale.

6 Cet ouvrage superbement illustré propose donc des pistes stimulantes à tous ceux qui s'intéressent au phénomène de la modernité, aux imaginaires et aux appropriations sociales des techniques, aux mobilités et aux usages de l'espace, au contrôle et au rapport entre l'homme et la machine.

\section{NOTES}

1. John Urry, Sociology beyond societies. Mobilities for the twenty-first Century, Londres, Routledge, 2000. 


\section{AUTEURS}

\section{LARISSA ZAKHAROVA}

Centre d'études des mondes russe, caucasien et centre-européen (CERCEC) 